\title{
Prolotherapy, Myths and the Truth
}

\section{Polukhin $\mathrm{E}^{*}$}

Rehabilitation Consultants, PA, CEO and Medical Director, Bush Medical Leadership Fellow, MN State Rehabilitation Council, USA

\begin{abstract}
Pain management has changed over the last 10 years. People seek medical attention that is safe, effective and yield success as documented by clinical practice and scientific studies.

This article is going to review on the comprehensive pain management program which known as prolotherapy, an injection technique that has been successfully used all over the world and is gaining popularity in the United States.
\end{abstract}

Keywords: Prolotherapy; Myo-fascial hypothesis; Musculo-skeletal painful conditions

\section{Introduction}

Pain management can be understood in terms of reduction in pain. It is the ability to reduce the pain which can be of any type [1].

Webster's Third New International Dictionary [2] defines prolotherapy as "the rehabilitation of an incompetence structure, such as a ligament or tendon by the induced proliferation of new cells [2]." This therapy technique involves an injection of substances to the area where ligaments and tendons attach to the bones. This creates a stimulating of the ligaments and tendons to proliferate or grow at the injection area. The area to which the injection is administered is called the fibro-osseous junction. "Fibro" stands for fibrous tissue that forms the ligament or tendon. "Osseous" refers to the bone.

The idea of prolotherapy is based on the myo-fascial hypothesis that bodily pain is caused primarily by ligamentous laxity, or enthesopathy. Painful muscle spasms are secondary and appear as late sequelae of ligamentous laxity, where treatment of the myo-fascial pain should be targeted to restore the tendon structure. Complete stabilization of tendon tissue will then naturally resolve the muscle spasms and the trigger points. This myo-fascial hypothesis was widely accepted in Europe, especially, in the East European countries and in the Olympic sports medical circles [3-5]. Generations of sports medicine physicians and medical providers specializing in musculo-skeletal pain held a firm belief that fixing the damaged, degenerated ligaments will cure the whole body pain and clinically result in overall pain control and functional enhancement [4-6].

I personally recall an experience from my early medical past that affirms the above-mentioned assertion. I remember how my PhD teacher in the Russian Olympic Committee Medical Commission made a demonstration of effects of torn ligaments and joints on patients using a rubber glove. My teacher said, "Good tendon should be soft and elastic like this glove. Any wrinkle or tendon tear can cause pain and functional decline. Take a good care of your athletes' tendons and ligaments." Today, such a simplistic demonstration would not pass the muster of any adequate medical school classroom. And while certainly such a simplistic approach is not accepted by practicing community, prolotherapy still has its role in comprehensive pain management today.

\section{Tendons and ligaments}

Ligaments are the structural "rubber bands" that hold the bones in place in our joints. Tendons connect the muscles to the bones. Both tendons and ligaments can become weaker because of age, injuries, and degenerative changes. There is a long list of factors that can potentially damage ligaments: lactic acidosis, heavy metals, stress, fatigue, narcotic medications, processed food, etc. Natural healing of tendons and ligaments is slow and most often than not, tendons and ligaments may not heal back to their original strength or endurance. Both ligament and tendons tissues have poor blood supply. Some scientists believe in a grown adult, the ligaments and tendons do not have any blood vessels whatsoever. Therefore the synthesis of collagen and other substances needed for tissue restoration is sluggish and limited.

At the same time, the nerve supply of tendons and ligaments is superior to other parts of the body. Many nerve endings signal the body regarding the function of the ligaments, the stretching of the ligaments, general muscle tension and body position while in movement or at rest. This is why even minimal structural damage of tendons and ligaments usually causes a lot of discomfort and triggers chronic muscle spasms and pain. Such pain can often radiate to arms and legs, resembling severe radicular pain.

The diagnosis of ligament laxity is an essential element of treating the problem. Diagnosis is usually made by examining the patient's complete history and performing careful physical examination with special provocative tests [5-9]. Reviewing image studies of the affected areas often aids in arriving at a proper diagnosis. Typically, a patient with ligamentous laxity often exhibits a history of chronic joint or spine pain exacerbated by any physical activity, limited therapeutic effect from simple analgesics and often limited response to physical therapy or manual manipulations. In fact, most patients with ligamentous laxity often complain of exacerbation of their pain after physical therapy and chiropractic manipulations. Such chronic pain might be a result of an acute or chronic injury, cumulative trauma disorder or a gradual development caused by professional activity that includes repetitive joint movements. Physical examination of such patients often reveals very tender focal areas over the painful site. While X-rays do not show the ligaments themselves, they are nonetheless necessary to rule out other sources of pain or limited range of motion.

*Corresponding author: Elena Polukhin, Rehabilitation Consultants, PA, CEO and Medical Director, Bush Medical Leadership Fellow, MN State Rehabilitation Council, USA, E-mail: elenapolukhin@hotmail.com

Received April 01, 2013; Accepted April 26, 2013; Published April 29, 2013

Citation: Polukhin E (2013) Prolotherapy, Myths and the Truth. Altern Integ Med 2: 114. doi: $10.4172 / 2327-5162.1000114$

Copyright: (c) 2013 Polukhin E. This is an open-access article distributed unde the terms of the Creative Commons Attribution License, which permits unrestricted use, distribution, and reproduction in any medium, provided the original author and source are credited. 


\section{Prolotherapy Approaches}

Prolotherapy was always attractive for patients and physicians. Prolotherapy promotes genuine, natural body healing and uses no artificial substances, no medications and nothing that can cause adverse future side effects. Prolotherapy method of treatment is simple: it requires an injection of proliferant solutions (dextrose, phenol, ethanol, etc.) into the tendon and ligament tissue [8-10]. Proliferative solutions cause local aseptic inflammation that triggers an increase in the blood supply. This process promotes nutrients flow to the tissue and an indirect stimulation of fibroblast activity, collagen growth and other bioactive substances synthesis. In other words, prolotherapy naturally promotes tissue rejuvenation.

On top of actually tissue rejuvenation, prolotherapy has a wonderful effect on the overall well being of the patient. Patients who are treated through this method report feeling a natural high, mood enhancement, extra energy and speedy recovery after only several sessions. While this phenomenon is difficult to explain scientifically, most specialists speculate that occurs due to a release of endorphins and other bioactive substances after skin and soft tissue penetration. Other specialists claim that inflammation induction leads to this euphoric feeling in their patients. This phenomenon is well known to prolotherapy practitioners and was widely utilized in top level sports in training sessions.

Historical review shows that a version of this technique was first used by Hippocrates on soldiers with dislocated, torn shoulder joints. Hippocrates, also known as the father of medicine, was said to stick a hot poker into the joint, leading to a miraculously fast healing results of the joint. Certainly today such clinical methods are not utilized, but the concept remains the same-the idea is that such stimulation should promote natural, aseptic local inflammation, trigger the natural selfrepair tissue mechanisms, innate ability that the body has and get the body to repair itself [11].

\section{Prolotherapy: Mechanism of Action}

"Prolo" stands for "proliferate." Basic scientific research demonstrates that injection of proliferative solutions causes a local aseptic inflammation, increase stem cells concentration in situ and accelerated fibroblasts transformation. Local collagen synthesis significantly increases and new tendon tissue is formatted as a result of activated structural proteins synthesis.

Prolotherapy sessions might enlarge the size of tendons and ligaments up to 40 percent $[6,8-10]$. It has also been shown that prolotherapy sessions increase the tendons fibrils strength by as much as 200 percent [10]. Scar tissue does not form as a result of this procedure and the new tissue that grows in place has been proven to be smooth, strong, flexible and highly functional.

Furthermore, additional research showed that prolotherapy sessions might dissolve post-surgical scars, rejuvenating poorly differentiated scar tissue and promote intracellular collagen synthesis. And while the exact mechanism of pain control is not clear, specialists speculate that because the intra-ligament nerve endings are no longer stretched and irritated, the end result is a better, less painful contact area.

The exact prolotherapy mechanisms of action are difficult to determine and demonstrate in the double controlled randomized studies. But lack of scientific evidence and unclear mechanisms of action is fate of many holistic procedures including acupuncture, manual manipulations and many other folk medicine techniques. Scientists argue about the mode of action and, and patients are paying big bucks for holistic medicine treatments in often questionable "medical offices". The great advantage of prolotherapy is that it is safe. Prolotherapy does not use any dangerous medications, reported local and generalized side effects are minimal and self resolving. Prolotherapy has existed in a physician's pain management armamentarium for ages, gaining popularity in many cultures all over the world.

\section{Disorders Traditionally Treated with Prolothrapy}

Prolotherapy can substantially alleviate many musculo-skeletal painful conditions including major and minor arthritis. These are some of the painful conditions that have been treated success chronic neck and low back pain, chronic muscle spasms, migraines, and other disorders caused by injured or weakened connective tissue, ligaments or tendons. List of several examples of conditions treated by Prolotherapy is given in table 1 .

Since then the prolotherapy indications significantly widened and enthusiastic physicians are constantly opening new horizons for this technique: hip dislocation, plantar fasciitis, rheumatoid arthritis, impotence, depression, alcoholism, drug addiction, aids neuropathy and many others.

\section{Prolotherapy Session}

Procedure for prolotherapy is usually conduced as follows. The first procedure is typically scheduled for the morning so the patient has sufficient time for recuperation after the injections. The usual session includes 20-30 injections of proliferative solution inside the tendons and ligaments in affected area (neck, shoulders, and knees). The low back area typically requires more injections, often as much as 50 on each side. The nurse positions the patient on the procedure table and sterilizes the area for injection with alcohol and iodine. Local anesthesia is used and often Lidocaine or similar anesthetics are applied on the patient's body. Most physicians mark the intended area of treatment identifying bony prominences, attachments of tendons and ligaments as well as tendon sheath. Thereafter, an injection takes place.

There are different techniques of Prolotherapy. Most physicians use the Hackett-Hemwell technique of injections [11]. Experienced practitioners develop several modification of the classic technique, but the principles of injections is the same-never inject the solution if you are not on the bone. According to classic prolotherapy procedures, the physician has to inject the bony-tendinous attachments or tendon sheath, so all the injections are done when the practitioner feels the bone. This procedure makes Prolotherapy safe because the possibility of penetrating life essential blood vessels or pleural cavity is very slim. Typical prolotherapy sessions last between 30 and 45 minutes however for back neck injections tend to take approximately one hour [9-11].

Overall, post injection pain is quite well tolerated by the patient. Post-injections pain is easily controlled by simple analgesics like Tylenol.

\begin{tabular}{|c|c|c|}
\hline Arthritis & Migraines & Sciatica \\
\hline Back Pain & Myofascial Pain Syndrome & Scoliosis \\
\hline Bone Spurs & Neck Pain & Slipping Rib \\
\hline Bunions & Osteoporosis & Spondylolisthesis \\
\hline Carpal Tunnel Syndrome & Plantar Fasciitis & Sports Injuries \\
\hline Fibromyalgia & Post Surgery Pain & Subluxation \\
\hline Herniated Discs & Pregnancy Back Pain & Tendonitis \\
\hline Ligament Sprains & RSD Pain & Tennis Elbow \\
\hline Loose Joints & Rotator Cuff Pain & TMJ Syndrome \\
\hline Meniscal Tear & Sacroiliac Laxity & ...and a host of others! \\
\hline
\end{tabular}

Table 1: List of conditions treated by Prolotherapy. 
The physician should discourage the patient to take anti-inflammatory medications immediately after the session. Because the general focus of Prolotherapy is to induce localized aseptic inflammation, antiinflammatory medications should be avoided within first 24-48 hours immediately following injections.

Good pain control is typically reached after several prolotherapy sessions. Some patients respond well to a single session, especially if the injection is administered to the smaller joints. Other patients, such as the elderly, patients with chronic degenerative spine changes, physical laborers, professional dancers and athletes requires about two or three treatments. In some cases, such patients require as many as eight treatments. As the healing progresses, the number of injections required per treatment decreases. In some cases, when the treatment does not yield quick results, and the patient continues to suffer from chronic pain, both the patient and physician may become frustrated. However, such frustration is easily avoided with setting reasonable expectations in both the patient and in the educated provider. Our experience shows that amount of collagen growth required for stabilization of the joint is different for each person. However, in due course, the therapeutic effect of Prolotherapy takes its course and the patient begins to heal. The important goal is to continue the Prolotherapy treatment. With continued treatment, there is an excellent chance of achieving total pain relief with the resumption of all previous activities, including athletics.

\section{Conclusion}

In the conclusion, I would like to emphasize that in no means I advocate for prolotherapy being a panacea from all musculo-skeletal pains. Being an old and respected method of alternative pain treatment, I think it has its place in comprehensive pain management programs. My experience shows that Prolotherapy as a solo modality might be not effective enough to completely alleviate tendons pain. However, when combined with an integrative pain management program, Prolotherapy is a valuable addition to traditional pain killers, manual manipulations, etc. I have been combining Prolotherapy with classic physical therapy, topical anesthetics, manual manipulations and acupuncture in treating chronic pain $[12,13]$. Any practicing physician should consider this treatment option in chronic pain patient, especially when other options provided limited effect, and the patient is eager to try non-traditional, complementary methods of treatment [12-15].

\section{References}

1. Asher A (2007) What is Pain Management? About.com Guide.

2. Webster's Third New International Dictionary.

3. Dagenais S, Yelland MJ, Del Mar C, Schoene ML (2007) Prolotherapy injections for chronic low-back pain. Cochrane Database Syst Rev CD004059.

4. Jansen JA, Mens JM, Backx FJ, Kolfschoten N, Stam HJ (2008) Treatment of longstanding groin pain in athletes: a systematic review. Scand J Med Sci Sports 18: 263-274.

5. Jensen KT, Rabago DP, Best TM, Patterson JJ, Vanderby R Jr (2008) Response of knee ligaments to prolotherapy in a rat injury model. Am J Sports Med 36: 1347-1357.

6. Fullerton BD (2008) High-resolution ultrasound and magnetic resonance imaging to document tissue repair after prolotherapy: a report of 3 cases. Arch Phys Med Rehabil 89: 377-385.

7. Hooper RA, Frizzell JB, Faris P (2007) Case series on chronic whiplash related neck pain treated with intraarticular zygapophysial joint regeneration injection therapy. Pain Physician 10: 313-318.

8. Wilkinson HA (2005) Injection therapy for enthesopathies causing axial spine pain and the "failed back syndrome": a single blinded, randomized and crossover study. Pain Physician 8: 167-173.

9. Linetsky F, Saberski L, Dubin JA, Miguel R, Wilkinson H (2004) Re: Yelland MJ Glasziou PP, Bogduk N, et al. Prolotherapy injections, saline injections, and exercises for chronic low-back pain: a randomized study. Spine. 2003;29:9-16 Spine 29: 1840-1841.

10. Reeves KD, Klein RG, DeLong WB (2004) Re: Yelland MJ, Glasziou PP Bogduk N, et al. Prolotherapy injections, saline injections, and exercises for chronic low-back pain: a randomized study. Spine. 2003;29:9-16. Spine 29: 1839-40.

11. Hackett GS (1960) Prolotherapy in whiplash and low back pain. Postgrad Med 27: $214-219$

12. Polukhin E (2007) Comprehensive pain management. Alternative view from the Alternative Clinic. Proceedings of the International Society of Physical and Rehabilitation Medicine. Monduzzi Publishers, Italy.

13. Polukhin E (2008) Intergrative Pain Management Program. Advances in Rehabilitation (in print).

14. Fleming S, Rabago DP, Mundt MP, Fleming MF (2007) CAM therapies among primary care patients using opioid therapy for chronic pain. BMC Complement Altern Med 7: 15

15. Staal JB, de Bie R, de Vet HC, Hildebrandt J, Nelemans $P$ (2008) Injection therapy for subacute and chronic low-back pain. Cochrane Database Syst Rev CD001824. 